\title{
Neutrino mixing and Lorentz invariance
}

\author{
Massimo Blasone ${ }^{\sharp}$, João Magueijo and Paulo Pires-Pacheco ${ }^{b}$ \\ \# Institute für Theoretische Physik, Freie Universität Berlin, Arnimallee 14, D-14195 Berlin, Germany \\ b Blackett Laboratory, Imperial College London, Prince Consort Road, London SW7 2BW, U.K.
}

\begin{abstract}
We use previous work on the Hilbert space for mixed fields to derive deformed dispersion relations for neutrino flavor states. We then discuss how these dispersion relations may be incorporated into frameworks encoding the breakdown of Lorentz invariance. We consider non-linear relativity schemes (of which doubly special relativity is an example), and also frameworks allowing for the existence of a preferred frame. In both cases we derive expressions for the spectrum and end-point of beta decay, which may be used as an experimental probe of the peculiar way in which neutrinos experience Lorentz invariance.
\end{abstract}

The subject of neutrino oscillations has now matured from an insightful prediction by Bruno Pontecorvo [1] and the early results of Homestake [2] to a structured framework backed by a wealth of new quantitative data [3-6]. These advances have been paralleled by much progress on the theoretical front, both in the phenomenological pursuit of more refined oscillation formulae and in efforts to give the theory a sound formal structure within Quantum Field Theory (QFT).

A major outstanding question is that of the existence of a Hilbert space for the flavor states [7]. Pontecorvo's treatment of flavor states as Quantum Mechanical superpositions is forbidden by the Bargmann super-selection rules [8] (see, however, Ref. [9]). This problem only found its resolution with a full QFT treatment [10-15]. In the resulting picture, flavor states - rather than mass eigenstates - constitute the real physical entities. On this basis oscillation formulae were derived which exhibit corrections with respect to the usual ones $[11,15,13,14]$.

In this letter we elaborate on the curious fact that neutrino flavor states don't satisfy the standard dispersion relations $E^{2}-k^{2}=m^{2}$, where $E$ is energy, $k$ momentum and $m$ rest mass. This follows trivially from the fact that flavor states are superpositions of mass eigenstates, which do satisfy standard dispersion relations, but with different masses.

Such a peculiarity leads to a formal connection between neutrino oscillations and a very different field. Deformed dispersion relations have been used as a phenomenological framework for quantum gravity [18-20], capable of explaining high-energy cosmic ray anomalies [21-24], and of establishing an observer independent border between the classical and quantum pictures of space-time [25-27].

Thus it is possible to employ a formalism describing violations of Lorentz invariance in quantum gravity to examine how neutrino flavor states must experience some form of breakdown of standard Lorentz invariance. Deformed dispersion relations signal either the presence of a preferred frame $[18,19]$ or a non-linear realization of Lorentz invariance [27] (for simplicity we exclude the possibility of quantum groups). We consider how neutrino flavor states might fit both possibilities, focusing mainly on the second.

We restrict ourselves to the simplest case, i.e. twoflavor mixing in the Pontecorvo approximation, and leave the full QFT treatment and the extension to three flavors to a longer publication [28]. We identify the nonlinear Lorentz transformations which leave the neutrino deformed dispersion relations frame-independent. We then use these results to work out energy conservation formulae, with and without a preferred frame. These lead to distinct predictions for the spectrum of beta decay, which may thus be used as a test of Lorentz invariance. We also discuss the meaning of a possible negative mass squared, as suggested by some observations [16,17].

Consider the mixing relations for two flavors:

$$
\begin{aligned}
& \nu_{e}(x)=\cos \theta \nu_{1}(x)+\sin \theta \nu_{2}(x) \\
& \nu_{\mu}(x)=-\sin \theta \nu_{1}(x)+\cos \theta \nu_{2}(x)
\end{aligned}
$$

Without loss of generality we take $0 \leq \theta \leq \frac{\pi}{4}$ and $m_{2}>m_{1}$. As explained in [10], it is possible to define a vacuum state, creation and annihilation operators, and a Hilbert space for flavor states. The properly defined flavor states $[11,15]$ are then eigenstates of the flavor charge and of the momentum operators ${ }^{1}$. Obviously they are not eigenstates of the Hamiltonian, however it makes sense to consider the expectation value of $H$ on the flavor states and define from it dispersion relations. In this paper we consider the simplest case, i.e. we limit ourselves to the usual Pontecorvo states, which are a good approximation for the full QFT flavor states when the masses are sufficiently close to each other. We then have

$$
\begin{aligned}
& \left|\nu_{e}\right\rangle=\cos \theta\left|\nu_{1}\right\rangle+\sin \theta\left|\nu_{2}\right\rangle \\
& \left|\nu_{\mu}\right\rangle=-\sin \theta\left|\nu_{1}\right\rangle+\cos \theta\left|\nu_{2}\right\rangle
\end{aligned}
$$

\footnotetext{
${ }^{1}$ For example, the electron neutrino state is defined by $Q_{e}\left|\nu_{e}\right\rangle=\left|\nu_{e}\right\rangle$ and $\mathbf{P}_{e}\left|\nu_{e}\right\rangle=\mathbf{k}\left|\nu_{e}\right\rangle$, with $Q_{e}=\int d^{3} x \nu_{e}^{\dagger}(x) \nu_{e}(x)$ and $\mathbf{P}_{e}=\int d^{3} \mathbf{x} \nu_{e}^{\dagger}(x)(-i \nabla) \nu_{e}(x)$.
} 
If we compute the expectation value of the Hamiltonian on the flavor states (2) we find the result:

$$
\begin{aligned}
& E_{e} \equiv\left\langle\nu_{e}|H| \nu_{e}\right\rangle=\omega_{k, 1} \cos ^{2} \theta+\omega_{k, 2} \sin ^{2} \theta \\
& E_{\mu} \equiv\left\langle\nu_{\mu}|H| \nu_{\mu}\right\rangle=\omega_{k, 2} \cos ^{2} \theta+\omega_{k, 1} \sin ^{2} \theta
\end{aligned}
$$

where $H\left|\nu_{i}\right\rangle=\omega_{i}\left|\nu_{i}\right\rangle(i=1,2)$ and

$$
\omega_{k, i}=\sqrt{\mathbf{k}^{2}+m_{i}^{2}} .
$$

Since the sum of two square roots is generally not a square root, we find that, except for trivial cases, flavor states do not satisfy the usual dispersion relations. Obviously the energies in Eq. (3) are only expectation values subject to fluctuations but it is nevertheless sensible to consider the modified Lorentz transformation for the classical limit of the theory.

It is immediately obvious that the minimal energy of a flavor state is achieved at zero momentum and is

$$
\begin{aligned}
& m_{e} \equiv E_{e}(\mathbf{k}=0)=m_{1} \cos ^{2} \theta+m_{2} \sin ^{2} \theta \\
& m_{\mu} \equiv E_{\mu}(\mathbf{k}=0)=m_{2} \cos ^{2} \theta+m_{1} \sin ^{2} \theta
\end{aligned}
$$

However this "mass" is not the kinematic invariant, since the dispersion relations Eq. (3) are no longer invariant under the usual Lorentz transformations. Instead, if we are to avoid introducing a preferred frame, neutrinos must feel a non-linear representation of the Lorentz group as constructed by $[27,24]$. The dispersion relations Eq. (3) may be put in the form used in that work:

$$
\begin{aligned}
E_{e}^{2} f_{e}^{2}\left(E_{e}\right)-\mathbf{k}^{2} g_{e}^{2}\left(E_{e}\right) & =M_{e}^{2} \\
E_{\mu}^{2} f_{\mu}^{2}\left(E_{\mu}\right)-\mathbf{k}^{2} g_{\mu}^{2}\left(E_{\mu}\right) & =M_{\mu}^{2}
\end{aligned}
$$

from which the recipes given in [24] are straightforward to apply. Eq. (3) leads to

$$
\begin{aligned}
& \left(E_{e}^{2}-\omega_{1}^{2} \cos ^{4} \theta-\omega_{2}^{2} \sin ^{4} \theta\right)^{2}=4 \omega_{1}^{2} \omega_{2}^{2} \sin ^{4} \theta \cos ^{4} \theta \\
& \left(E_{\mu}^{2}-\omega_{2}^{2} \cos ^{4} \theta-\omega_{1}^{2} \sin ^{4} \theta\right)^{2}=4 \omega_{1}^{2} \omega_{2}^{2} \sin ^{4} \theta \cos ^{4} \theta
\end{aligned}
$$

and, for example, Eq. (9) can be rewritten as a quadratic equation in $\mathbf{k}^{2}$, of the form $A \mathbf{k}^{4}+B \mathbf{k}^{2}+C=0$, with

$$
\begin{aligned}
& A=\cos ^{2} 2 \theta \\
& B=2 \cos (2 \theta) \tilde{m}_{-}^{2}-E_{e}^{2}\left(1+\cos ^{2} 2 \theta\right) \\
& C=E_{e}^{4}-2 E_{e}^{2} \tilde{m}_{+}^{2}+\tilde{m}_{-}^{4}
\end{aligned}
$$

and $\tilde{m}_{ \pm}^{2}=m_{1}^{2} \cos ^{4} \theta \pm m_{2}^{2} \sin ^{4} \theta$. Assuming first that $\theta \neq \pi / 4$, its solutions are

$$
\begin{aligned}
\mathbf{k}_{ \pm}^{2} & =\frac{E_{e}^{2}\left(1+\cos ^{2} 2 \theta\right)-2 \tilde{m}_{-}^{2} \cos 2 \theta \pm \sqrt{\Delta}}{2 \cos ^{2} 2 \theta} \\
\Delta & =E_{e}^{2} \sin ^{4} 2 \theta\left(E_{e}^{2}+\left(m_{2}^{2}-m_{1}^{2}\right) \cos 2 \theta\right)
\end{aligned}
$$

Since $E$ is a monotonically growing function of $k$ with minimum $m_{e}$, the discriminant satisfies $\Delta>0$, so that the roots are guaranteed to be real. The physically relevant solution is $\mathbf{k}_{-}^{2}$ (e.g. study the special case $m=m_{1}=m_{2}$ ). Comparing (14) and (7) leads to:

$$
\begin{aligned}
2 f_{e}^{2}\left(E_{e}\right)= & 1+\frac{1}{\cos ^{2}(2 \theta)} \\
& -\frac{\sqrt{E_{e}^{2}+\left(m_{2}^{2}-m_{1}^{2}\right) \cos (2 \theta)}}{E_{e}} \tan ^{2}(2 \theta) \\
g_{e}^{2}\left(E_{e}\right)= & 1 \\
M_{e}^{2}= & \frac{\tilde{m}_{-}^{2}}{\cos 2 \theta}=\frac{m_{1}^{2} \cos ^{4} \theta-m_{2}^{2} \sin ^{4} \theta}{\cos (2 \theta)}
\end{aligned}
$$

Notice that $M_{e}^{2}<0$ for

$$
\tan \theta>\sqrt{m_{1} / m_{2}}
$$

This does not imply causality violations, since it can be shown (directly from Eq. (3)) that the neutrino velocity (defined either as $v=p / E$ or as $v=d E / d p$ ) is a monotonically growing function of its momentum, with $v<1$. However other aspects of tachyonic behavior will be identified later.

The case of maximal mixing $\theta=\pi / 4$ has to be treated separately. Then $A=0$ in Eq. (11), collapsing the order of the equation. One then finds trivially

$$
\begin{aligned}
f_{e}^{2}\left(E_{e}\right) & =1+\left(\frac{m_{1}^{2}-m_{2}^{2}}{4 E_{e}^{2}}\right)^{2} \\
g_{e}^{2}\left(E_{e}\right) & =1 \\
M_{e}^{2} & =\frac{m_{1}^{2}+m_{2}^{2}}{2}
\end{aligned}
$$

Similar expressions, but with $m_{1} \leftrightarrow m_{2}$, apply to muon neutrinos. In the whole parameter space $M_{\mu}^{2}>0$.

It is now possible to identify the non-linear realization of the Lorentz group which leaves these dispersion relations invariant. They are generated by the transformation $U \circ(E, \mathbf{k})=(E f, \mathbf{k} g)$ applied to the standard Lorentz generators $\left(L_{a b}=p_{a} \frac{\partial}{\partial p^{b}}-p_{b} \frac{\partial}{\partial p^{a}}\right)$ :

$$
K^{i}=U^{-1}\left[p_{0}\right] L_{0}^{i} U\left[p_{0}\right] .
$$

This amounts to requiring linearity for the auxiliary variables $\tilde{E}=E f(E)$ and $\tilde{k}=k g(E)$. The resulting nonlinear transformations for $E$ and $k$ are a non-linear representation of the Lorentz group ensuring that the deformed dispersion relations found for flavor states are valid in all frames. For $\nu_{e}$ the transformation (23) only exists for

$$
E_{e}^{2} \geq E_{\text {min }}^{2}=\left(m_{2}^{2}-m_{1}^{2}\right) \frac{\sin ^{4} 2 \theta}{4 \cos 2 \theta}
$$

However, if $M_{e}^{2} \geq 0$, one has $E_{\min }<E_{e}(k=0)=m_{e}$, so that the transformation exists for all allowed values of $E_{e}$. This is not the case if $M_{e}^{2}<0$. Then one must impose $|k|>k_{\min }=\sqrt{-M_{e}^{2}}$ to have a well defined transformation (23). Since this condition is frame-independent, no 
conflict with the principle of relativity arises (notice also that $E \geq 0$ always transforms into $E \geq 0$ ).

The ugly alternative to non-linear realizations is that neutrino flavor states are subject to the usual linear Lorentz transformations, so that their deformed dispersion relations select a preferred frame (typically the cosmological frame). With or without the introduction of a preferred frame, flavor states are at odds with conventional Lorentz invariance.

We now explore the physical implications of these peculiarities, taking as an example beta decay: $A \rightarrow$ $B+e^{-}+\bar{\nu}_{e}$ where $A$ and $B$ are two nuclei (e.g. ${ }^{3} \mathrm{H}$ and ${ }^{3} \mathrm{He}$ ). Given that the nuclei and the electron satisfy linear Lorentz transformations, and that $E_{e} f_{e}\left(E_{e}\right)$ transforms linearly (cf. Eq. (23)), the only covariant law of energy conservation is

$$
E_{A}=E_{B}+E+E_{e} f_{e}\left(E_{e}\right) .
$$

where $E$ is the electron energy, and as before the subscript $e$ refers to the electron neutrino. If, on the contrary, we insist upon the standard law

$$
E_{A}=E_{B}+E+E_{e}
$$

we have introduced a preferred frame, and are in conflict with the principle of relativity.

These two choices are reflected in different predictions for the endpoint of $\beta$ decay, that is, the maximal kinetic energy $\left(K_{\max }\right)$ the electron can carry away. This is constrained by the available energy $Q=E_{A}-E_{B}-m \approx$ $m_{A}-m_{B}-m$, where $m$ is the electron mass. For the tritium decay, $Q=18.6 \mathrm{KeV}$. $Q$ is shared between the (unmeasured) neutrino energy and the (measured) electron kinetic energy $K$. If the neutrino were massless, then $K_{\max }=Q$. If the neutrino were a mass eigenstate (say with $m_{e}=m_{1}$ ), then $K_{\max }=Q-m_{1}$.

Under the effects of flavor mixing, the answer depends crucially on how flavor states conflict with Lorentz invariance. If there is a preferred frame in nature (and Eq. (26) is valid) then

$$
K_{\text {max }}=Q-m_{e}=Q-\left(m_{1} \cos ^{2} \theta+m_{2} \sin ^{2} \theta\right)
$$

since the minimal neutrino energy is given by Eq. (5). The spectrum is proportional to the phase volume factor $E p E_{e} p_{e}$, so that

$$
\frac{d N}{d K}=C E p(Q-K) \sqrt{(Q-K)^{2} f_{e}^{2}(Q-K)-M_{e}^{2}}
$$

where $E=m+K$ and $p=\sqrt{E^{2}-m^{2}}$ are the electron's energy and momentum. Here $C$ is a constant and we have neglected the Coulomb interaction between the final particles. We have illustrated this possibility in Fig. 1 (for clarity we have chosen wildly unrealistic parameters).

However if we reject the existence of preferred frames, the statement of energy conservation must be Eq. (25). Then, if $M_{e}^{2} \geq 0$, the endpoint of $\beta$ decay is $K_{\max }=Q-$

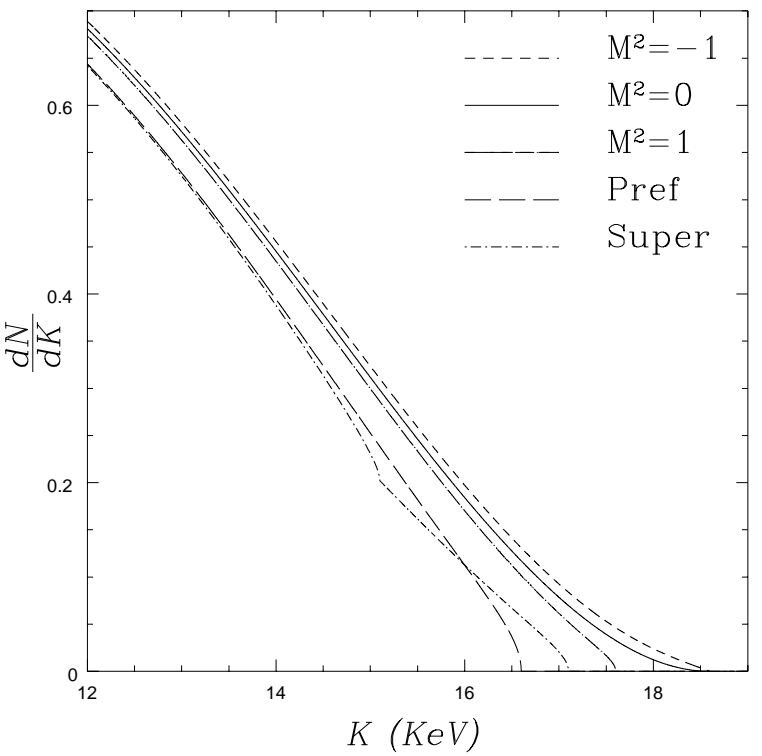

FIG. 1. The tail of the tritium $\beta$ spectrum for a massless neutrino (solid) and for Lorentz invariant flavor states with $M_{e}^{2}= \pm 1 \mathrm{KeV}^{2}$ (dashed and dot-long dashed lines). We have also plotted the case of neutrinos with a preferred frame and $m_{1}=1.5 \mathrm{KeV}, m_{2}=3.5 \mathrm{KeV}, \theta=30^{\circ}$ (for which $M_{e}=1$ $\mathrm{KeV}$ but $m_{e}=2 \mathrm{KeV}$ ) and the superposed prediction for 2 mass states with the same parameters (notice the inflexion in the spectrum where the most massive state switches off).

$M_{e}$, since the minimum of $E_{e} f_{e}\left(E_{e}\right)$ is $M_{e}$, the kinematic invariant defined by Eq. (7). The $\beta$ spectrum is now proportional to the phase volume factor $E p E_{e} f_{e}\left(E_{e}\right) p_{e}$, so that:

$$
\frac{d N}{d K}=C E p(Q-K) \sqrt{(Q-K)^{2}-M_{e}^{2}}
$$

in contradiction with Eq. (28). The only measurable parameter is now $M^{2}$, and in Fig. 2 we plotted the contours in $\left\{\tan (\theta), m_{1} / m_{2}\right\}$ space along which the likelihood is aligned. This is to be contrasted with oscillation experiments, which are sensitive to $\theta$ and $\Delta m^{2}=m_{2}^{2}-m_{1}^{2}$. We have also plotted in Fig.1 predictions for $M_{2}^{2}=0, \pm 1$ $\mathrm{KeV}$. We see that the tail of the spectrum is distinctly different from the case where there is a preferred frame.

The case $M_{e}^{2}<0$ merits special consideration. It does not imply faster than light propagation, or the need for a preferred frame. Upon closer inspection, however, we find that $E_{e} f_{e}\left(E_{e}\right)$ is not bounded from below. Furthermore, imposing the condition $E_{e} f_{e}\left(E_{e}\right) \geq 0$ (in the same way that we have imposed $k>k_{\min }$ before) violates Lorentz invariance. Thus we obtain an unstable theory (with $K_{\max }=\infty$ ) unless we are prepared to accept preferred frames (or a maximal boost parameter for any given frame).

Thus, at the level of interactions, the case $M_{e}^{2}<0$ has to violate the principle of relativity. The minimum of $E f(E)$ is zero, so that the endpoint of $\beta$ decay is $K_{\max }=$ $Q$. Eq. (29) is still valid, and in this case one observes 


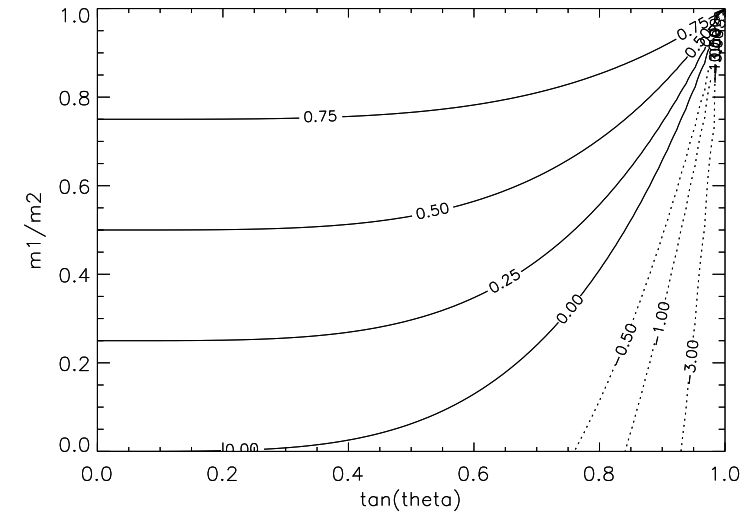

FIG. 2. Contours of the parameter $M^{2}$, upon which $\beta$ decay depends $\left(M^{2}\right.$ in units of $\left.m_{2}^{2}\right)$. The likelihood of any decay experiment should follow these lines in $\left\{\tan (\theta), m_{1} / m_{2}\right\}$ space.

an excess (rather than a deficiency) of events near the endpoint, as compared to the zero mass case. This seems currently to be favored by observations $[16,17]$.

The limiting case $M_{e}=0$, is on the contrary consistent with the principle of relativity. Such a neutrino does not behave like a massless particle: it travels slower than light, and has a rest frame (unlike the case $M_{e}^{2}<0$ ). One can impose the condition $E_{\nu} f_{\nu}\left(E_{\nu}\right) \geq 0$ without violating Lorentz invariance. So the end point of $\beta$ decay is $K_{\max }=Q$, and Eq. (29) is valid, so that the zero mass, no-mixing case is perfectly mimicked as far as $\beta$ decay is concerned.

To conclude, all these predictions are significantly different from those obtained by giving primacy to the mass (rather than flavour) eigenstates [29]. Then the $\beta$ spectra is

$$
\frac{d N}{d K}=C E p E_{e} \sum_{i}\left|U_{e i}\right|^{2} \sqrt{E_{e}^{2}-m_{i}^{2}} \Theta\left(E_{e}-m_{i}\right)
$$

(where $E_{e}=Q-K$ and $U_{e i}=(\cos \theta, \sin \theta)$ ). The end point is at $K=Q-m_{1}$ and the spectrum has an inflexion at $K=Q-m_{2}$. We have also plotted this possibility in Fig. 1.

In summary, we have investigated how flavor states cannot satisfy standard dispersion relations, and studied the implications for the principle of relativity. We found that although we may introduce a preferred frame to describe these states, this is not necessary as long as we are prepared to consider non-linear realizations of the Lorentz group. The only exception is the region of parameter space defined by Eq. (19), where, contrary to all appearances, one needs a preferred frame to enforce stability.

Having laid down all possibilities, we then computed the spectrum and endpoint of $\beta$ decay. We found a distinct prediction in each case, a matter of great interest given prospective experimental improvements [29]. Thus the unusual interplay between Lorentz invariance and neutrino flavor mixing is an issue to be decided by experimentalists.

Acknowledgements We thank K. Baskerville for help in connection with this paper. M. B. thanks the ESF network COSLAB and EPSRC for support.

[1] S. M. Bilenky and B. Pontecorvo, Phys. Rep. 41 (1978) 225 .

[2] J. Davis, D. S. Harmer and K. C. Hoffmann, Phys. Rev. Lett. 20 (1968) 1205.

[3] S. Fukuda et al. (Super-Kamiokande collaboration), Phys. Rev. Lett. 86 (2001) 5656.

[4] Q. R. Ahmad et al. (SNO collaboration), Phys. Rev. Lett. 87 (2001) 071301; Phys. Rev. Lett. 89 (2002) 011301.

[5] K. Eguchi et al. [KamLAND Collaboration], Phys. Rev. Lett. 90 (2003) 021802.

[6] M. H. Ahn et al. [K2K Collaboration], Phys. Rev. Lett. 90 (2003) 041801.

[7] C. Giunti, C. W. Kim and U. W. Lee, Phys. Rev. D 45 (1992) 2414. C. W. Kim and A. Pevsner, Neutrinos in Physics and Astrophysics, (Harwood Ac. Press, 1993).

[8] V. Bargmann, Annals Math. 59 (1954) 1; A. Galindo and P. Pascual, Quantum Mechanics, (Springer, 1990).

[9] D. M. Greenberger, Phys. Rev. Lett. (2001).

[10] M. Blasone and G. Vitiello, Annals Phys. 244 (1995) 283 [Erratum-ibid. 249 (1995) 363].

[11] M. Blasone, P. A. Henning and G. Vitiello, Phys. Lett. B 451 (1999) 140.

[12] K. C. Hannabuss and D. C. Latimer, J. Phys. A 33 (2000) 1369; J. Phys. A 36 (2003) L69.

[13] M. Binger and C. R. Ji, Phys. Rev. D 60 (1999) 056005; C. R. Ji and Y. Mishchenko, Phys. Rev. D 64 (2001) 076004; Phys. Rev. D 65 (2002) 096015.

[14] K. Fujii, C. Habe and T. Yabuki, Phys. Rev. D 59 (1999) 113003; Phys. Rev. D 64 (2001) 013011.

[15] M. Blasone, A. Capolupo, O. Romei and G. Vitiello, Phys. Rev. D 63 (2001) 125015; M. Blasone, A. Capolupo and G. Vitiello, Phys. Rev. D 66 (2002) 025033; M. Blasone, P. Jizba and G. Vitiello, Phys. Lett. B 517 (2001) 471; M. Blasone, P. P. Pacheco and H. W. Tseung, Phys. Rev. D 67 (2003) 073011; M. Blasone and J. S. Palmer, [hep-ph/0305257].

[16] A. I. Belesev et al., Phys. Lett. B 350 (1995) 263.

[17] V. M. Lobashev et al., Phys. Lett. B 460 (1999) 227.

[18] G. Amelino-Camelia et al., Int. J. Mod. Phys. A 12 (1997) 607; G. Amelino-Camelia et al., Nature 393 (1998) 763.

[19] J. Ellis et al., Astrophys. J. 535 (2000) 139.

[20] J. Ellis, N. E. Mavromatos and D. Nanopoulos, Phys. Rev. D 63 (2001) 124025; ibidem [astro-ph/0108295].

[21] P. Biermann and G. Sigl, L. Notes Phys. 576 (2001) 1.

[22] M. Takeda et al., Astrophys. J. 522 (1999) 225; Phys. Rev. Lett. 81 (1998) 1163.

[23] G. Amelino-Camelia and T. Piran, Phys. Rev. D 64 (2001) 036005. 
[24] J. Magueijo and L. Smolin, Phys. Rev. D 67 (2003) 044017.

[25] G. Amelino-Camelia, Int. J. Mod. Phys. D 11 (2002) 35; [gr-qc/0012051]; G. Amelino-Camelia, Phys. Lett. B 510 (2001) 255.

[26] J. Kowalski-Glikman, Phys. Lett. A 286 (2001) 391; N. R. Bruno, G. Amelino-Camelia, J. Kowalski-Glikman, Phys. Lett. B 522 (2001) 133.

[27] J. Magueijo and L. Smolin, Phys. Rev. Lett. 88 (2002) 190403.

[28] M. Blasone, J. Magueijo and P. Pires-Pacheco, preprint.

[29] A. Osipowicz et al., [hep-ex/010903]. 